\title{
3 Research Square

\section{Genetic Chinese Medicine Network Pharmacology Research, Quercetin Improves Drug Resistance in Acute Myeloid Leukemia by Targeting CXCL10}

Hu Linjun

Zhejiang Provincial People's Hospital

QILIANG LU

Qingdao University Medical College

YANG LIU

Qingdao University Medical College

JUNJUN ZHAO

Bengbu Medical College

ZHI ZENG

Qingdao University Medical College

ZHAN SHI

Zhejiang Chinese Medical University

YIFENG TU

Zhejiang Chinese Medical University

ZUNQIANG XIAO

Zhejiang Chinese Medical University

QIURAN XU ( $\nabla$ windway626@sina.com )

Zhejiang Provincial People's Hospital

XIANGMIN TONG

Zhejiang Provincial People's Hospital

Research

Keywords: Acute myeloid leukemia, network pharmacology, quercetin, CXCL10

Posted Date: March 1st, 2021

DOI: https://doi.org/10.21203/rs.3.rs-258594/v1

License: (1) (1) This work is licensed under a Creative Commons Attribution 4.0 International License.

Read Full License 


\section{Abstract}

Background: Acute myeloid leukemia (AML) is the most common acute leukemia in adults and is a highly heterogeneous and fatal disease. At present, the main method of treatment of AML is chemotherapy, but patients who relapse often develop resistance and are not sensitive to chemotherapy. Chinese medicine network pharmacology can provide new ideas about improving AML resistance.

Methods: The gene expression data of relapsed drug-resistant AML and primary AML are from Gene Expression Omnibus (GEO) database. Based on the network pharmacology of traditional Chinese medicine, the effective components and target genes of Jiedu Huayu Decoction were analyzed. we performed Kyoto Encyclopedia of Genes and Genomes (KEGG), Gene Ontology (GO) analyses, Proteinprotein interaction (PPI) network and construction on overlapping genes. We will perform prognostic analysis and gene correlation analysis of overlapping genes in GEPIA. The binding energy between the differential gene and the active ingredient of the drug was studied by molecular docking.

Results: We found that quercetin, the active ingredient in Jiedu Huayu Decoction, can target CXCL 10, thereby improving AML resistance.

Conclusions: In this study, we found that quercetin improves drug resistance in acute myeloid leukemia by targeting CXCL 10 based on the GEO database and the network pharmacology study of Chinese medicine.

\section{Introduction}

Acute myeloid leukemia (Acute myeloid leukemia, AML) is a type of malignant hematological disease characterized by rapid progression of the disease and poor prognosis[1]. According to the latest cancer statistics in 2020, there have been 11,180 deaths and 19,940 new cases of AML[2]. With the improvement of chemotherapy regimens, hematopoietic stem cell transplantation, gene targeted therapy, biological immunotherapy, traditional Chinese medicine and other treatment methods, some progress has been made in the treatment of AML. However, due to severe side effects and multi-drug resistance, about twothirds of the patients are prone to relapse after first-line treatment $[3,4]$. After recurrence, chemotherapy has poor effect and poor prognosis, and there is no good treatment plan for the patients after recurrence $[5,6]$. Once AML patients relapse, the tumor cells are easy to develop resistance to chemotherapeutic drugs, and it is easy to develop multi-drug resistance to a variety of chemotherapeutic drugs with different structures and functions, and then it is difficult to cure[7-9]. Therefore, there is an urgent need to develop new drugs to improve AML resistance.

Traditional Chinese Medicine (TCM) has been used in Asia for more than 2,000 years. Due to its outstanding efficacy, abundant resources and low toxicity, it has gradually been accepted by non-Chinese medicine practitioners. In the clinical treatment of cancer, many traditional Chinese medicine formulations are used alone or as adjuvants for conventional chemotherapy[10,11]. Studies have shown that Chinese medicine combined with chemotherapy can increase the remission rate of AML patients and 
improve the resistance of $A M L$ patients[12, 13]. The jiedu huayu recipe medicine is composed of Indigo Naturalis, Pseudobulbus Cremastrae Seu Pleiones, Paris polyphylla, Polygonum cuspidatum, Curcuma Zedoaria, Ligusticum chuanxiong Hort, Salvia Miltiorrhiza and Psoraleae Fructus. Studies have shown that this prescription has an important effect on improving drug resistance in leukemia patients[14]. In traditional Chinese medicine formulations, a variety of herbal ingredients and biologically active ingredients target multiple receptors and produce synergistic or antagonistic effects[15]. However, its drug components are numerous and drug interactions are complex, which brings certain difficulties to the mechanism of its anti-tumor effect. Chinese medicine network pharmacology is a new research field that combines pharmacology and pharmacodynamics. It is based on systems biology, multi-directional pharmacology and high-throughput analysis, analyzes the connection between drugs, targets and diseases by building a biological network, and has been widely used in Chinese medicine to treat cancer[16, 17]. Analyzing the ingredients of Jiedu Huayu Recipe through network pharmacology, we discovered a hydroxyflavonoid called quercetin, which is the active ingredient of Polygonum cuspidatum and its chemical name is 3,3,4,5,7 -Pentahydroxyflavone[18]. Anti-inflammatory, antioxidant and anticancer activities are some of the mainly described quercetin mechanisms of action[19-21]. And it has been found that quercetin can improve the remission rate of AML chemotherapy and induce apoptosis of HL60 cells[22, 23]. Studies have found that quercetin can improve the drug resistance of prostate cancer, breast cancer, gastric cancer and other cancers[24-26], but its mechanism of improving drug resistance in AML is less.

In this study, we searched for the bioactive ingredients and targets of the drug through the network pharmacology of traditional Chinese medicine and the GEO database to screen AML relapsed resistance genes, and finally found that quercetin targets CXCL 10 and cooperates with HMOX1 and IRF1 improve AML resistance.

\section{Materials And Methods}

\section{Microarray data}

We extracted gene expression (GSE66525) profiling data from the Gene Expression Omnibus (GEO) database at the National Center for Biotechnology Information. The AML-associated dataset GSE66525 submitted by Wieser R based on the GPL11532 platform was obtained from the GEO database and includes $11 \mathrm{AML}$ primary samples and $11 \mathrm{AML}$ recurrence normal samples.

\section{Identification of differentially expressed genes (DEGs)}

The limma package is a core component of Bioconductor, an R-based open-source software development project in statistical genomics[27]. The package is designed in such a way that, after initial preprocessing and normalization, the same analysis pipeline is used for data from all technologies. For the data from GEO, the R package limma was applied to perform analysis to identify the DEGs. The "R" software was applied to construct heat maps and Volcano map, and the regions in which the differential genes were mainly concentrated were highlighted. 
All components of the eight Chinese medicinal herbs in jiedu huayu recipe (Indigo Naturalis, Pseudobulbus Cremastrae Seu Pleiones, Paris polyphylla, Polygonum cuspidatum, Curcuma Zedoaria, Ligusticum chuanxiong Hort, Salvia Miltiorrhiza and Psoraleae Fructus) were retrieved from the traditional Chinese medicine systems pharmacology (TCMSP) database (http://tcmspw.com/)[28]. In drug absorption, distribution, metabolism, and excretion (ADME) processes, oral bioavailability (OB) is one of the most significant pharmacokinetic parameters[29]. As a qualitative concept applied in drug design to estimate the druggability of a molecule[30], the drug-likeness (DL) index is useful for rapid screening of active substances. The active components were the filtered by combining oral bioavailability $(O B) \geq 30 \%$ and drug-likeness (DL) index $\geq 0.18$ as suggested by the TCMSP database.

\section{Prediction of Drug Targets for Biologically active ingredients in jiedu huayu recipe}

The protein targets of the active substances in jiedu huayu recipe were retrieved from the TCMSP database and the traditional Chinese medicine integrated database (TCMID, http://www.megabionet.org/tcmid/).

\section{Screening of overlapping genes between DEGs and drug targets of biologically active ingredients}

The target gene of the active ingredient of the drug and the differential gene in the GEO database are analyzed by Perl software, and the overlapping part of the two differential genes is defined as a common differential gene, and its expression level in the GEO database is stored for subsequent analysis.

\section{Functional enrichment analyses for overlapping genes}

The Kyoto Encyclopedia of Genes and Genomes (KEGG) is a database resource for understanding highlevel functions and utilities of the biological system from molecular-level information. The Gene Ontology (GO) could be used to perform enrichment analysis. We used DAVID (https://david.ncifcrf.gov/) to make KEGG pathway analysis and GO enrichment analysis.

\section{Protein-protein interaction (PPI) network construction}

PPI analysis is used to search core genes and gene modules related to carcinogenesis. In this study, PPI network analysis of the overlapping genes were performed using the search tool for the Retrieval of Interacting Genes (STRING) database.

\section{Prognostic Analysis and Gene Correlation Analysisin GEPIA}

Gene Expression Profiling Interactive Analysis (GEPIA), a web-based tool to deliver fast and customizable functionalities based on TCGA and GTEx data, provides key interactive and customizable functions including differential expression analysis, profiling plotting, correlation analysis, patient survival analysis, similar gene detection and dimensionality reduction analysis[31]. We used GEPIA to analyze the prognosis of 17 overlapping genes. P-values $<0.05$ were considered statistically significant. Then we used 
prognostic-related genes and 17 overlapping genes for genetic correlation analysis. The correlation of gene expression was evaluated by Spearman's correlation and statistical significance, and the strength of the correlation was determined using R $\otimes 0.5$.

\section{Molecular docking}

Molecular docking is a key tool in structural molecular biology and computer-assisted drug design[32]. We use molecular docking to predict the combination of active ingredients of drugs and differential genes. First of all, we prepared 3D structure of targets and compounds which were mined from Protein Data Bank (PDB) (https://www.rcsb.org/) and PubChem (https://pubchem.ncbi.nlm.nih.gov/) databases[33, 34]. Then, the AutoDock tool was applied to perform a molecular docking[35]. Finally, PyMOL 2.3.2 software was used for visual processing to check the binding status of ligands and receptor binding sites[36].

\section{Results}

\section{Identification of differentially expressed genes in primary and relapsed AML patients}

For the AML-associated dataset GSE66525 from GEO, the R package limma was applied to perform analysis to identify the DEGs. We screened a total of 458 differential genes, of which 133 genes were upregulated in patients with relapsed $A M L$, and 326 genes were down-regulated in patients with recurrent AML. Heat map and volcano map show differential gene expression (Fig. 1A, 1B).

\section{Network pharmacological analysis of Chinese medicine effective ingredients and targets of Jiedu Huayu Recipe}

We first screened the effective ingredients of eight Chinese herbal medicines(Indigo Naturalis, Pseudobulbus Cremastrae Seu Pleiones, Paris polyphylla, Polygonum cuspidatum, Curcuma Zedoaria, Ligusticum chuanxiong Hort, Salvia Miltiorrhiza and Psoraleae Fructus) in the Jiedu Huayu Recipe in the TCMSP database by setting the conditions of $O B \geq 30 \%$ and $D L \geq 0.18$, and then searched for the target genes corresponding to each active ingredient. We found the active ingredients of six kinds of traditional Chinese medicines in the TCMSP database, but did not find the active ingredients of Paris polyphylla and Psoraleae Fructus. When searching for the target genes of the active ingredients, we found five of the target genes of traditional Chinese medicine, but did not find the target of the active ingredients of Pseudobulbus Cremastrae Seu Pleiones. We organize the drugs, active ingredients, and target genes into Table 1. 
Table 1

Drug active ingredients and target genes corresponding to the prescription drugs in Jiedu Huayu Recipe.

$\begin{array}{lll}\text { Drug } & \begin{array}{l}\text { Active } \\ \text { ingredients }\end{array} & \text { Target gene }\end{array}$

Salvia

MOL007101 NCOA2

Miltiorrhiza

MOL007088 NCOA2

MOL007093 PPARG, NCOA2

MOL007064 NCOA2

MOL007049 PPARG, NCOA2

MOL007122 NCOA2

MOL007098 NCOA2

MOL007041 PPARG, NCOA2

MOL007143 NCOA2

MOL007119 NCOA2

MOL007036 NCOA2

MOL007108 NCOA2

MOL007115 NCOA2

MOL002651 PPARG

MOL007125 PPARG, NCOA2

MOL007132 PPARG

MOL007100 PPARG

MOL007050 PPARG

MOL007154 CASP3

MOL001771 NCOA2

MOL007120 NCOA2

MOL001601 NCOA2

MOL007156 PPARG, NCOA2

MOL000006 NCOA2, MMP2, CASP3, PPARG, HMOX1, IFNGR1, PTGES

MOL000358 NCOA2, CASP3, PRKCA 


\begin{tabular}{|c|c|c|}
\hline Drug & $\begin{array}{l}\text { Active } \\
\text { ingredients }\end{array}$ & Target gene \\
\hline \multirow{6}{*}{$\begin{array}{l}\text { Ligusticum } \\
\text { Chuanxiong } \\
\text { Hort }\end{array}$} & MOL002157 & NCOA2 \\
\hline & MOL002135 & PPARG \\
\hline & MOL001494 & NCOA2 \\
\hline & MOL000359 & NCOA2 \\
\hline & MOL000006 & NCOA2, MMP2, CASP3, PPARG, HMOX1, IFNGR1 PTGES \\
\hline & MOL000358 & NCOA2, CASP3, PRKCA \\
\hline \multirow{3}{*}{$\begin{array}{l}\text { Indigo } \\
\text { Naturalis }\end{array}$} & MOL002309 & PPARG \\
\hline & MOL000006 & NCOA2, MMP2, CASP3, PPARG, HMOX1, IFNGR1, PTGES \\
\hline & MOL000358 & NCOA2, CASP3, PRKCA \\
\hline \multirow{6}{*}{$\begin{array}{l}\text { Polygonum } \\
\text { Cuspidatum }\end{array}$} & MOL002268 & NCOA2 \\
\hline & MOL000492 & NCOA2 \\
\hline & MOL000098 & PPARG, NCOA2, MMP2, CASP3, PRKCA, HMOX1, CAV1, CYP1B1, \\
\hline & (Quercetin) & SERPINEI, IFINGRI, NCFI, PPARD, CXCL IU, SPPI, ACPP, IRFI \\
\hline & MOL000006 & NCOA2, MMP2, CASP3, PPARG, HMOX1, IFNGR1, PTGES \\
\hline & MOL000358 & NCOA2, CASP3, PRKCA \\
\hline \multirow{3}{*}{$\begin{array}{l}\text { Curcuma } \\
\text { Zedoaria }\end{array}$} & MOL000296 & NCOA2 \\
\hline & MOL000006 & NCOA2, MMP2, CASP3, PPARG, HMOX1, IFNGR1, PTGES \\
\hline & MOL000358 & NCOA2, CASP3, PRKCA \\
\hline
\end{tabular}

\section{Screening and analysis of overlapping genes between DEGs and bioactive ingredient drug targets}

We use Perl software to analyze the overlapping genes of the target gene of the active ingredient of the drug and the differential gene in the GEO database. We screened 17 overlapping genes. Compared with primary patients with $\mathrm{AML}$, the expression levels of overlapping genes in relapsed patients are sorted into Table 2. And we show the active ingredients of drugs corresponding to overlapping genes (Fig. 2A). We found that the 17 overlapping genes screened were basically consistent with the target genes of 
quercetin, the active ingredient of Polygonum cuspidatum (Table 3, Fig. 2B). We speculate that quercetin has a certain therapeutic effect on patients with relapsed resistant AML.

Table 2

The overlapping genes of the target gene of the active ingredient of the drug and the differential gene in the GEO database.

\begin{tabular}{|lll|}
\hline Genes & logFC & $p$ value \\
\hline NCOA2 & -0.71039374 & 0.019247061 \\
\hline PPARG & -0.824038153 & 0.048075407 \\
\hline MMP2 & 1.763107463 & 0.014933989 \\
\hline CASP3 & 0.830909926 & 0.010240489 \\
\hline HMOX1 & -1.524949264 & 0.010362237 \\
\hline IFNGR1 & -0.639788165 & 0.020564584 \\
\hline PTGES & -0.680890169 & 0.001482936 \\
\hline PRKCA & -1.240566198 & 0.037672849 \\
\hline CAV1 & 1.997562161 & 0.007925876 \\
\hline CYP1B1 & -1.981788198 & 0.007133341 \\
\hline SERPINE1 & 1.216215851 & 0.004921618 \\
\hline NCF1 & -1.719297665 & 0.008906454 \\
\hline PPARD & -0.75128786 & 0.017145662 \\
\hline CXCL10 & -1.771414628 & 0.006680325 \\
\hline SPP1 & 1.223800376 & 0.032785076 \\
\hline ACPP & -1.413469603 & 0.000649293 \\
\hline IRF1 & -0.722719669 & 0.000622327 \\
\hline
\end{tabular}

Table 3

\section{Classification Genes}

MOL000098 PPARG, NCOA2, MMP2, CASP3, PRKCA, HMOX1, CAV1, CYP1B1, SERPINE1, IFNGR1, (Quercetin) NCF1, PPARD, CXCL10, SPP1, ACPP, IRF1

Overlapping genes
PPARG, NCOA2, MMP2, CASP3, PRKCA, HMOX1, CAV1, CYP1B1, SERPINE1, IFNGR1, NCF1, PPARD, CXCL10, SPP1, ACPP, IRF1, PTGES 
Then, we constructed a PPI network with 17 overlapping genes, and performed KEGG pathway analysis (Fig. 2C, 2D). KEGG pathway analysis found that overlapping genes are mainly enriched in AGE-RAGE signaling pathway in diabetic complications, HIF-1 signaling pathway, Fluid shear stress and atherosclerosis, Influenza A, Proteoglycans in cancer, MicroRNAs in cancer, TNF signaling pathway, Leukocyte transendothelial migration, Osteoclast differentiation and Natural killer cell mediated cytotoxicity. Finally, GO analysis was performed with overlapping genes. Regarding the biological processes (BP), the overlapping genes were significantly enriched in response to lipopolysaccharide, response to molecule of bacterial origin, regulation of angiogenesis, response to oxygen levels, regulation of vasculature development, regulation of response to wounding, response to nutrient, negative regulation of cell migration, negative regulation of cell motility and epithelial cell migration (Fig. 2E). The molecular function (MF) enriched by the overlapping genes included ligand-dependent nuclear receptor transcription coactivator activity, peptidase regulator activity, transcription coactivator activity, long-chain fatty acid binding, RNA polymerase II transcription factor activity, sequence-specific transcription regulatory region DNA binding, transcription factor activity, sequence-specific DNA binding transcription factor recruiting, fatty acid binding, transcription coactivator activity, transcription factor recruiting, peptidase activator activity, nuclear receptor activity (Fig. 2F). In the cellular component (CC), the analysis revealed that enrichment mainly occurred at membrane raft, membrane microdomain, membrane region, caveola, plasma membrane raft (Fig. 2G).

\section{Correlation analysis and prognostic analysis of overlapping genes in GEPIA}

We used the GEPIA database to analyze whether 17 overlapping genes are related to the prognosis of patients with AML recurrence (Supplementary Fig. 1). P value $<0.05$ was considered statistically significant. We found that the CXCL10 gene is associated with the prognosis of patients with relapsed AML (Fig. 3A). We found that CXCL 10 is the target of quercetin, and we speculate that quercetin can improve the prognosis of patients with relapsed drug-resistant AML. Compared with primary AML patients, CXCL10 expression is low in relapsed patients, and low CXCL10 expression is an independent predictor of poor prognosis in relapsed patients. Then we used $C X C L 10$ to analyze the correlation with 17 overlapping genes, and found that HMOX1, IRF1 and CXCL10 have a high correlation (R >0.5) (Fig. 3B, 3C).

\section{Molecular docking}

The structure of $C X C L 10$ and quercetin were introduced into AutoDockTools for molecular docking. In general, the lower the binding free energy, the more stable the binding between the ligand and protein receptor. According to the results of molecular docking, we found that quercetin has a strong binding activity with $C X C L 10$ (Fig. 4).

\section{Discussion}


Acute myeloid leukemia (AML) is the most common acute leukemia in adults and is a highly heterogeneous and fatal disease[37]. At present, the main method of treatment of AML is chemotherapy, but patients who relapse often develop resistance and are not sensitive to chemotherapy. Finding new therapeutic drugs and targets is the key to improving drug resistance. In this study, we analyzed the active ingredients and targets of the drug in Jieduhuayu Decoction based on the network pharmacology of Chinese medicine, and analyzed the differential genes between patients with relapsed drug-resistant AML and patients with primary AML through the GEO dataset to find methods to improve AML resistance.

This study is the first bioinformatics study to report the relationship between TCM network pharmacology and AML relapsed drug resistance differential genes. Through the differential gene screening of Chinese medicine network pharmacology and GEO database, we finally found 17 coincident genes, and found that 16 of these 17 coincident genes are the target genes of quercetin. By analyzing 17 overlapping genes, we found that $C X C L 10$ is related to the prognosis of AML patients with relapsed resistance. Low expression of $C X C L 10$ in patients with relapsed resistance indicates a poor prognosis. And through the correlation analysis of 17 overlapping genes, we found that HMOX1, IRF1 and CXCL 10 have a good correlation. Therefore, we believe that quercetin affects the prognosis of patients with AML relapsed resistance by targeting CXCL 10 .

CXC chemokine ligand 10 (CXCL 10), also known as interferon-y-inducible protein 10 (IP-10), is one of the CXC chemokine superfamily, which can regulate immune response, angiogenesis, Apoptosis and other effects are related to the occurrence, development, treatment, and prognosis of various tumors[38, 39]. Studies have shown that $C X C L 10$ is related to cancer prognosis. Breast cancer patients with high $C X C L 10$ expression have a higher survival rate than breast cancer patients with low CXCL10 expression. CXCL 10 can be used as a beneficial predictor of tamoxifen in the treatment of breast cancer[40]. Research on rectal cancer shows that the high expression of CXCL10 has high treatment sensitivity, and CXCL 10 is related to the efficacy and prognosis of rectal cancer[41]. Studies on tongue cancer have shown that the expression of $C X C L 10$ has a significant correlation with the efficacy of radiotherapy for tongue cancer. The higher the expression of $C X C L 10$, the better the efficacy, which can be used as an evaluation index for the prognosis and overall survival rate of radiotherapy for tongue cancer[42]. Combined with previous studies and the results of our bio-analysis, we have reason to believe that targeting CXCL10 with quercetin can improve the prognosis of AML patients. Heme oxygenase-1 (HMOX1), as a key protein in oxidative stress, is related to the degradation of heme into biliverdin, iron and carbon monoxide[43]. Studies have found that HMOX1 is one of the most important mechanisms leading to AML chemotherapy resistance, and HMOX1 can inhibit cell apoptosis by activating the JNK/c-JUN signaling pathway[44]. Studies have found that HMOX1 can regulate the expression of $C X C L 10$ in colon cancer[45]. Interferon regulatory factor (IRF), an important type of transcriptional regulator, has a variety of functions in the regulation of gene expression during inflammation, immune response, cell proliferation, cell cycle progression, T cell differentiation and DNA damage[46]. Studies have shown that the up-regulation of IRF1 in cancer foci is considered to be a way to improve the prognosis and reduce the resistance to immunotherapy; and it has been found that IRF-1 response genes can reduce the risk of AML[47]. Some studies have shown that IRF1 plays a role in the corneal innate immune response by regulating the 
expression of CXCL10[48]. Combined with our research findings, HMOX1, IRF1 and CXCL 10 have a high correlation in AML patients. We speculate that HMOX1 and IRF1 may participate in CXCL10 to improve the prognosis of AML patients with relapsed drug resistance. At the same time, we also found that HMOX1, IRF1 and CXCL10 are all target genes of quercetin.

This study had several limitations: (1) the relatively small number of cases evaluated; (2) The pathway of the study has not been validated in vivo and in vitro experiments.

\section{Conclusion}

In summary, through a comprehensive analysis of gene expression and Chinese medicine network pharmacology, we have identified multiple abnormally expressed genes and pathways that may be related to $A M L$ relapse and drug resistance.

\section{Declarations}

Ethical Approval and Consent to participate

Not applicable

\section{Availability of data and materials}

Two gene expression datasets (GSE66525) that support the findings of this study are available in Gene Expression Omnibus (GEO) at [https://www.ncbi.nlm.nih.gov/geo/query/acc.cgi?acc=GSE66525].

\section{Acknowledgements}

This study was supported by Key Laboratory of Tumor Molecular Diagnosis and Individualized Medicine of Zhejiang Province, Zhejiang Provincial People's Hospital (Affiliated Hospital of Hangzhou Medical College).

\section{Funding}

This study was supported by grants from the National Science and Technology Major Project for New Drug (No. 2017ZX301033)

\section{Competing interests}

The authors declared that they have no competing interests.

\section{Authors' contributions}

LINJUN HU, QILIANG LU, YANG LIU, and JUNJUN ZHAO conceived the project and wrote the manuscript. ZHI ZENG, ZHAN SHI, and ZUNQIANG XIAO participated in data analysis. QIURAN XU participated in 
discussion and language editing. XIONGMIN TONG reviewed the manuscript.

$1 \#, 2 \#, 3 \#$, ZHI ZENG ${ }^{2}$, , QIURAN XU ${ }^{1}$ XIANGMIN TONG ${ }^{1}$

\section{Consent for publication}

The authors agreed to publish the manuscript.

\section{References}

1. Nagler, E., M.F. Xavier, and N. Frey, Updates in immunotherapy for acute myeloid leukemia. Translational Cancer Research, 2017. 6(1): p. 86-92.

2. Siegel, R., K. Miller, and A.J.C.a.c.j.f.c. Jemal, Cancer statistics, 2020. 2020. 70(1): p. 7-30.

3. Ferrara, et al., Acute myeloid leukaemia in adults. 2013.

4. Dohner, K., P. Paschka, and H. Dohner, [Acute myeloid leukemia]. Internist (Berl), 2015. 56(4): p. 35463.

5. Burnett, A., M. Wetzler, and B. Lowenberg, Therapeutic Advances in Acute Myeloid Leukemia. Journal of Clinical Oncology, 2011. 29(5): p. 487-494.

6. Shaffer, B.C., et al., Drug resistance: Still a daunting challenge to the successful treatment of AML. Drug Resistance Updates, 2012. 15(1-2): p. 62-69.

7. Ding, L., et al., Clonal evolution in relapsed acute myeloid leukaemia revealed by whole-genome sequencing. Nature, 2012. 481(7382): p. 506-510.

8. Kronke, J., et al., Clonal evolution in relapsed NPM1-mutated acute myeloid leukemia. Blood, 2013. 122(1): p. 100-8.

9. Zhang, J., Y. Gu, and B.A. Chen, Mechanisms of drug resistance in acute myeloid leukemia. Oncotargets and Therapy, 2019. 12: p. 1937-1945.

10. Eloranta, S., et al., How can we make cancer survival statistics more useful for patients and clinicians: an illustration using localized prostate cancer in Sweden. Cancer Causes Control, 2013. 24(3): p. 505-15.

11. Chernysh, S. and I. Kozuharova, Anti-tumor activity of a peptide combining patterns of insect alloferons and mammalian immunoglobulins in naive and tumor antigen vaccinated mice. Int Immunopharmacol, 2013. 17(4): p. 1090-3.

12. Ma, H.Y., et al., Ethyl acetate extract of Caesalpinia sappan L. inhibited acute myeloid leukemia via ROS-mediated apoptosis and differentiation. Phytomedicine, 2020. 68: p. 153142.

13. Wang, M., et al., Anti-angiogenic activity of ShengMaBieJia decoction in vitro and in acute myeloid leukaemia tumour-bearing mouse models. Pharm Biol, 2020. 58(1): p. 454-464.

14. Ma, W.K., Y.Y. Li, and X.M. Yao, [Effect of jiedu huayu recipe containing serum on the expressions of survivin and NF-kappaB of K562/A02 cell apoptosis by]. Zhongguo Zhong Xi Yi Jie He Za Zhi, 2011. 31(8): p. 1097-100. 
15. Zhou, X., et al., Current Status and Major Challenges to the Safety and Efficacy Presented by Chinese Herbal Medicine. Medicines (Basel), 2019. 6(1).

16. H, Y., et al., How Can Synergism of Traditional Medicines Benefit from Network Pharmacology? 2017. 22(7).

17. Chen, Y., et al., Eyes on systems pharmacology. Pharmacol Res, 2016. 114: p. 39-41.

18. Kawabata, K., R. Mukai, and A. Ishisaka, Quercetin and related polyphenols: new insights and implications for their bioactivity and bioavailability. Food Funct, 2015. 6(5): p. 1399-417.

19. Sharma, A., et al., Therapeutic charm of quercetin and its derivatives: a review of research and patents. Pharm Pat Anal, 2018. 7(1): p. 15-32.

20. Reyes-Farias, M. and C. Carrasco-Pozo, The Anti-Cancer Effect of Quercetin: Molecular Implications in Cancer Metabolism. Int J Mol Sci, 2019. 20(13).

21. Carullo, G., et al., Quercetin and derivatives: useful tools in inflammation and pain management. Future Med Chem, 2017. 9(1): p. 79-93.

22. Naimi, A., et al., Quercetin sensitizes human myeloid leukemia KG-1 cells against TRAIL-induced apoptosis. J Cell Physiol, 2019. 234(8): p. 13233-13241.

23. Lee, W.J., et al., Quercetin induces mitochondrial-derived apoptosis via reactive oxygen speciesmediated ERK activation in HL-60 leukemia cells and xenograft. Archives of Toxicology, 2015. 89(7): p. $1103-1117$.

24. Tummala, R., et al., Quercetin Targets hnRNPA1 to Overcome Enzalutamide Resistance in Prostate Cancer Cells. Mol Cancer Ther, 2017. 16(12): p. 2770-2779.

25. Hyun, H.B., J.Y. Moon, and S.K. Cho, Quercetin Suppresses CYR61-Mediated Multidrug Resistance in Human Gastric Adenocarcinoma AGS Cells. Molecules, 2018. 23(2).

26. Wang, H., et al., Quercetin reverses tamoxifen resistance in breast cancer cells. J BUON, 2015. 20(3): p. 707-13.

27. Gentleman, R.C., et al., Bioconductor: open software development for computational biology and bioinformatics. Genome Biol, 2004. 5(10): p. R80.

28. Ru, J., et al., TCMSP: a database of systems pharmacology for drug discovery from herbal medicines. J Cheminform, 2014. 6: p. 13.

29. Xu, X., et al., A novel chemometric method for the prediction of human oral bioavailability. Int J Mol Sci, 2012. 13(6): p. 6964-82.

30. Tao, W., et al., Network pharmacology-based prediction of the active ingredients and potential targets of Chinese herbal Radix Curcumae formula for application to cardiovascular disease. J Ethnopharmacol, 2013. 145(1): p. 1-10.

31. Tang, Z., et al., GEPIA: a web server for cancer and normal gene expression profiling and interactive analyses. Nucleic Acids Res, 2017. 45(W1): p. W98-W102.

32. Saikia, S. and M. Bordoloi, Molecular Docking: Challenges, Advances and its Use in Drug Discovery Perspective. Current Drug Targets, 2019. 20(5): p. 501-521. 
33. Burley, S.K., et al., Protein Data Bank (PDB): The Single Global Macromolecular Structure Archive. Methods Mol Biol, 2017. 1607: p. 627-641.

34. Wang, Y., et al., PubChem BioAssay: 2017 update. Nucleic Acids Res, 2017. 45(D1): p. D955-D963.

35. Jiang, X., et al., DOVIS 2.0: an efficient and easy to use parallel virtual screening tool based on AutoDock 4.0. Chem Cent J, 2008. 2: p. 18.

36. Mooers, B.H.M., Shortcuts for faster image creation in PyMOL. Protein Sci, 2020. 29(1): p. 268-276.

37. Wingelhofer, B. and T.C.P. Somervaille, Emerging Epigenetic Therapeutic Targets in Acute Myeloid Leukemia. Front Oncol, 2019. 9: p. 850.

38. Karin, N. and H. Razon, Chemokines beyond chemo-attraction: CXCL 10 and its significant role in cancer and autoimmunity. Cytokine, 2018. 109: p. 24-28.

39. Karin, N., Chemokines and cancer: new immune checkpoints for cancer therapy. Curr Opin Immunol, 2018. 51: p. 140-145.

40. Hilborn, E., et al., C-X-C ligand 10 and $C-X-C$ receptor 3 status can predict tamoxifen treatment response in breast cancer patients. 2014. 145(1): p. 73-82.

41. Li, C., et al., CXCL10 mRNA expression predicts response to neoadjuvant chemoradiotherapy in rectal cancer patients. Tumour Biol, 2014. 35(10): p. 9683-91.

42. Rentoft, M., et al., Expression of CXCL 10 is associated with response to radiotherapy and overall survival in squamous cell carcinoma of the tongue. 2014. 35(5): p. 4191-8.

43. Yang, Y.C., et al., Carbon monoxide induces heme oxygenase-1 to modulate STAT3 activation in endothelial cells via S-glutathionylation. PLoS One, 2014. 9(7): p. e100677.

44. Lin, X., et al., Heme oxygenase-1 suppresses the apoptosis of acute myeloid leukemia cells via the JNK/c-JUN signaling pathway. Leuk Res, 2015. 39(5): p. 544-52.

45. Seo, G.S., et al., Heme oxygenase-1 promotes tumor progression and metastasis of colorectal carcinoma cells by inhibiting antitumor immunity. Oncotarget, 2015. 6(23): p. 19792-806.

46. Alsamman, K. and O.S. El-Masry, Interferon regulatory factor 1 inactivation in human cancer. Biosci Rep, 2018. 38(3).

47. Willman, C.L., et al., Deletion of IRF-1, mapping to chromosome 5q31.1, in human leukemia and preleukemic myelodysplasia. Science, 1993. 259(5097): p. 968-71.

48. Yoon, G.S., et al., Interferon regulatory factor-1 in flagellin-induced reprogramming: potential protective role of CXCL 10 in cornea innate defense against Pseudomonas aeruginosa infection. Invest Ophthalmol Vis Sci, 2013. 54(12): p. 7510-21.

\section{Figures}


A

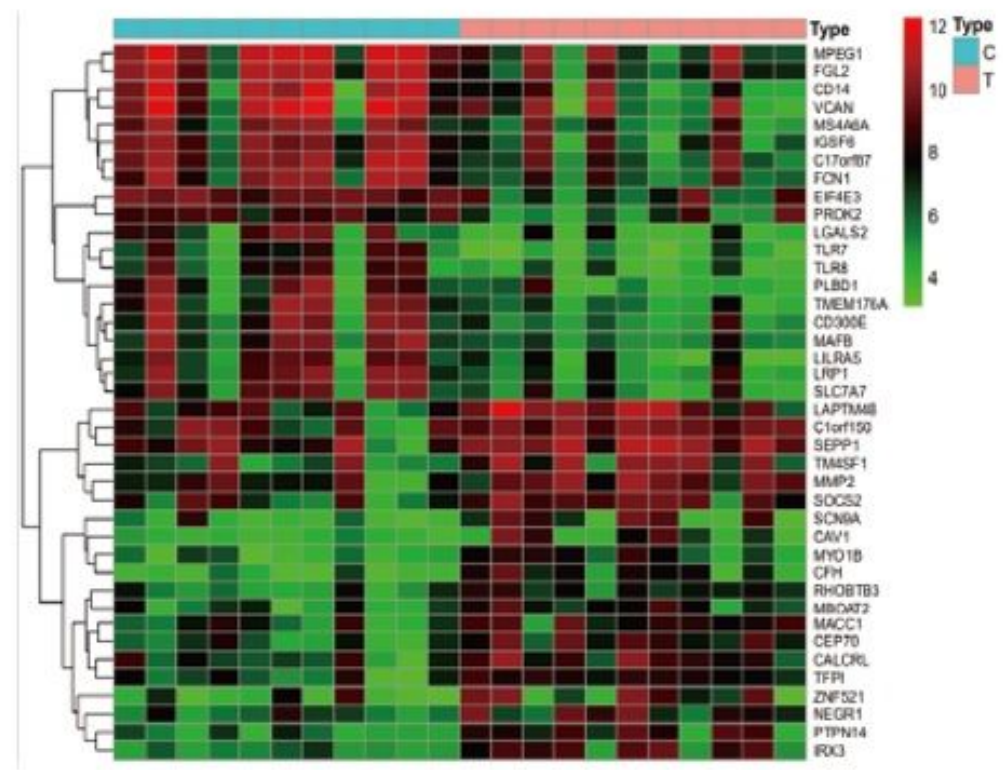

B

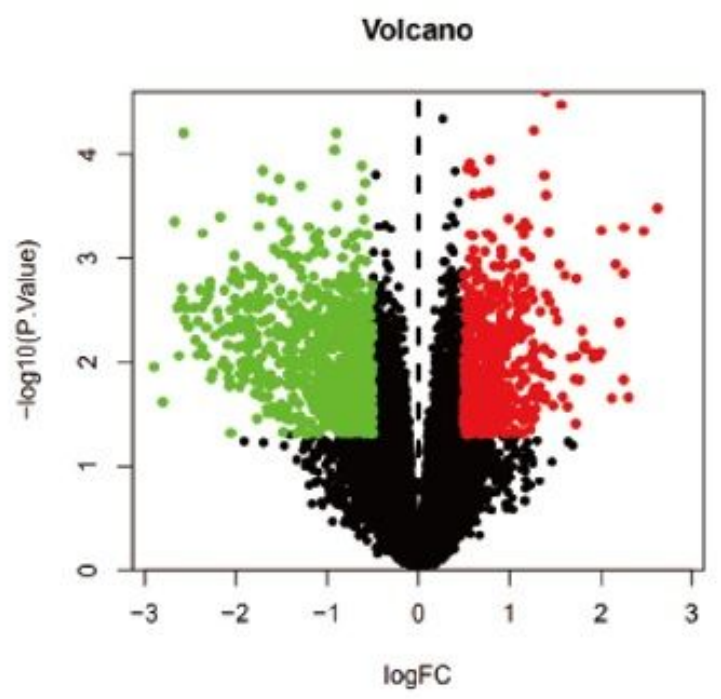

Figure 1

Identification of differentially expressed genes in gene expression datasets (GSE66525). (A) Heat map; (B) Volcano map. 

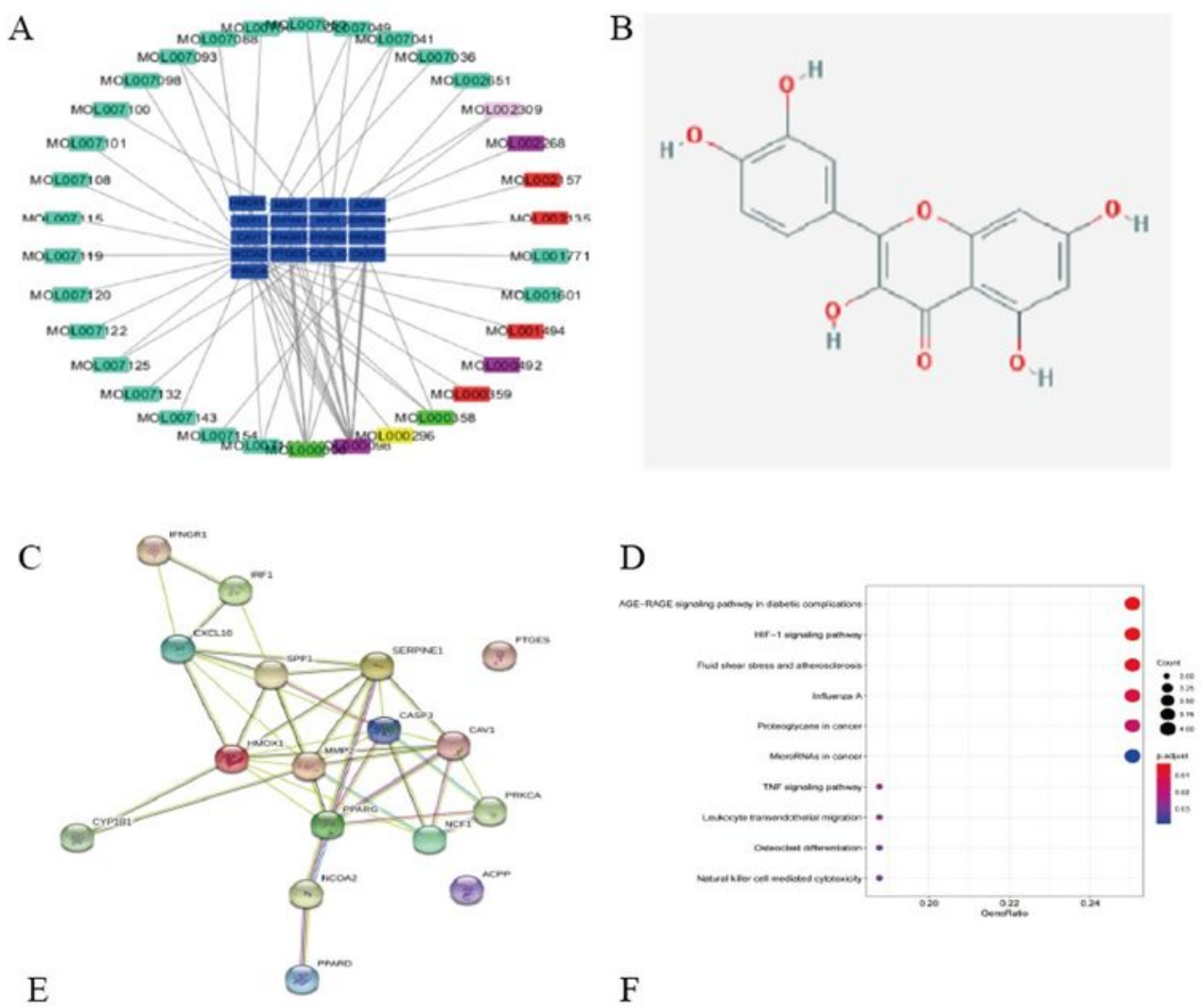

$\mathrm{E}$

F
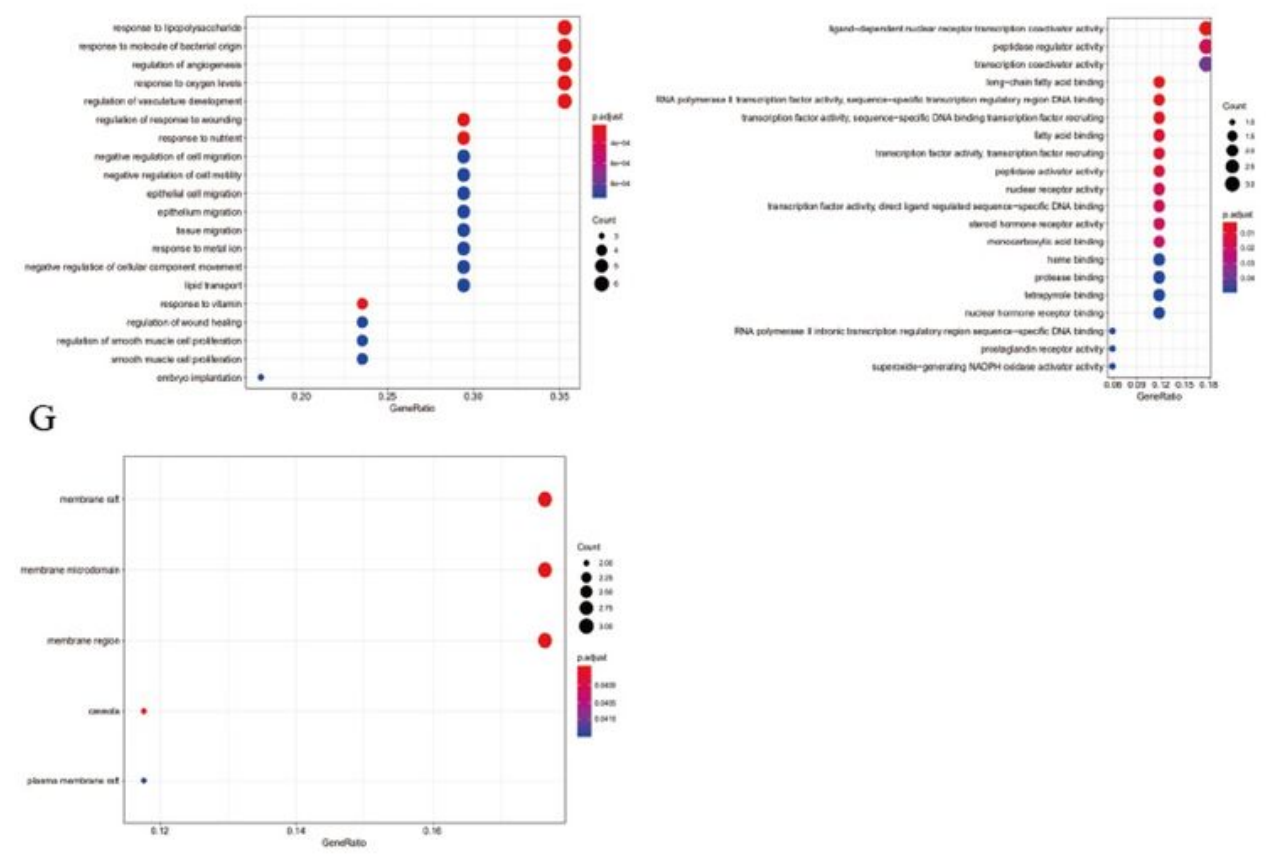

\section{Figure 2}

17 overlapping genes. (A) The active ingredients of drugs corresponding to overlapping genes; (B) Quercetin molecular formula; (C) PPI network of 17 overlapping genes; (D) KEGG pathway analysis; (E) GO analysis: the biological processes (BP); (F) GO analysis: the molecular function (MF); (G) GO analysis:the cellular component (CC). 
A

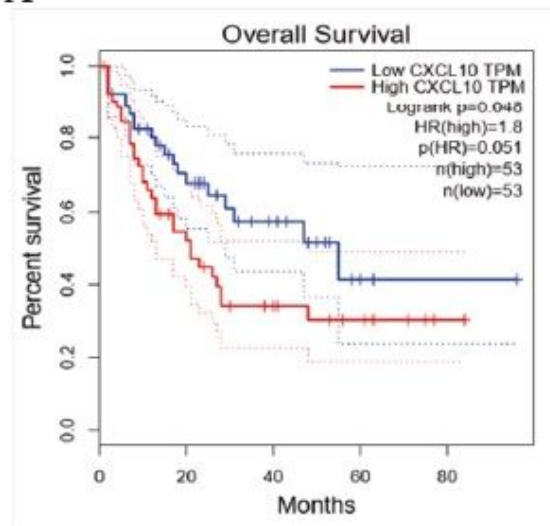

B

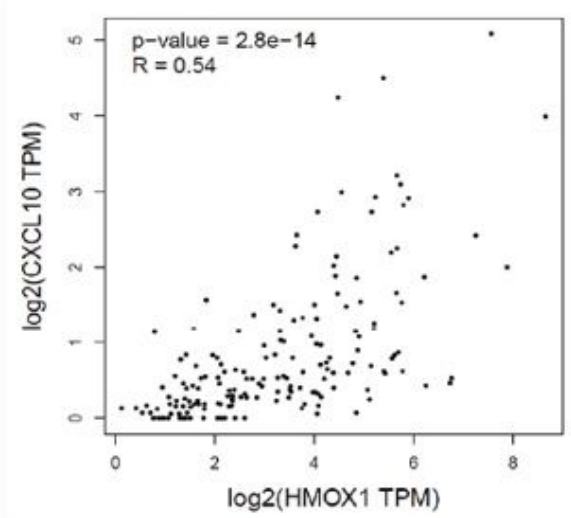

$\mathrm{C}$

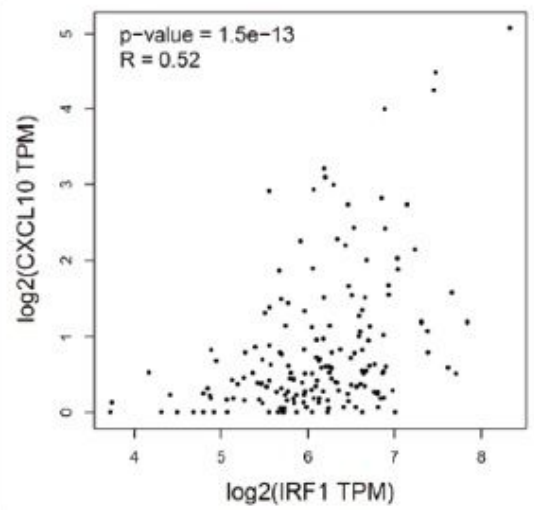

Figure 3

Correlation analysis and prognostic analysis of overlapping genes in GEPIA. (A) Prognosis of CXCL10; (B)Correlation analysis between CXCL10 and HMOX1; (C) Correlation analysis between CXCL10 and IRF1.
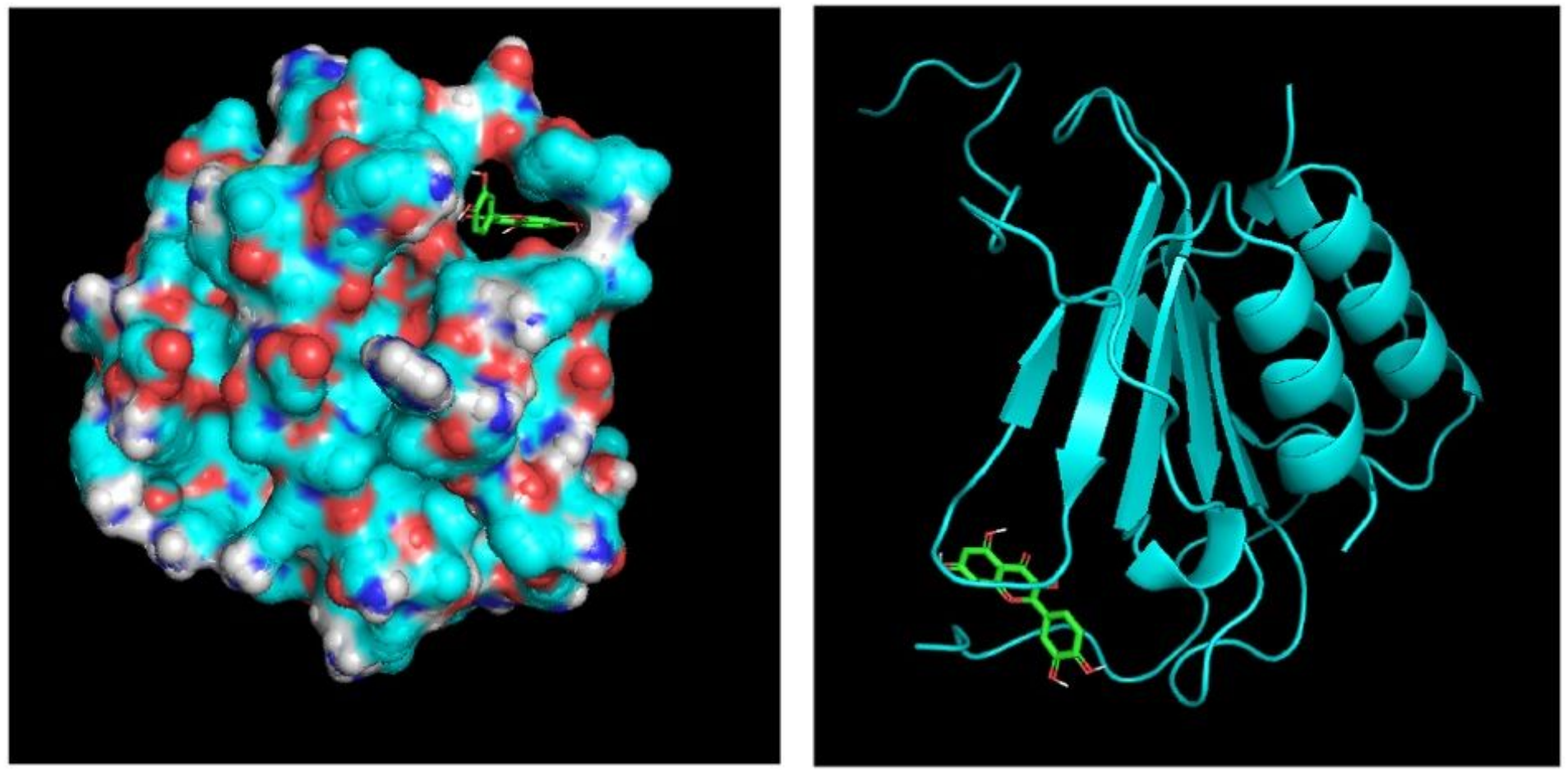

Figure 4

Molecular docking of CXCL10 with quercetin.

\section{Supplementary Files}

This is a list of supplementary files associated with this preprint. Click to download. 
- Supplement1.docx

- supplementaryGraphicalabstract.docx 